\title{
Characterization of thick film poly(triarylamine) semiconductor diodes for direct x-ray detection
}

\author{
Akarin Intaniwet, ${ }^{1, a)}$ Christopher A. Mills, ${ }^{1, a)}$ Maxim Shkunov, ${ }^{2}$ Heiko Thiem, ${ }^{3}$ \\ Joseph L. Keddie, ${ }^{1, b), c)}$ and Paul J. Sellin ${ }^{1, b), d)}$ \\ ${ }_{1}^{1}$ Department of Physics, University of Surrey, Guildford, Surrey GU2 7XH, United Kingdom \\ ${ }^{2}$ Advanced Technology Institute, University of Surrey, Guildford, Surrey GU2 7XH, United Kingdom \\ ${ }^{3}$ Evonik Degussa GmbH, Paul-Baumann-Str. 1, 45772 Marl, Germany
}

(Received 5 June 2009; accepted 17 August 2009; published online 28 September 2009)

Thick film ( $\geq 5 \mu \mathrm{m}$ thick) semiconducting polymer diodes incorporating poly(triarylamine) (PTAA) have been produced and applied as direct x-ray detectors. Experiments determined that a rectifying diode behavior persists when increasing the thickness of the active layer above typical thin film thicknesses $(<1 \mu \mathrm{m})$, and the electrical conduction mechanism of the diodes has been identified. Direct current and photoconductivity measurements on indium tin oxide/ poly(3,4-ethylenedioxythiophene)/poly(styrenesulfonate)/PTAA/metal diodes confirm that carrier conduction occurs via a Poole-Frenkel mechanism. The energy band structure of diodes (having gold or aluminum top electrodes) has been elucidated and used to explain the resulting electrical characteristics. Theoretical calculations show that, upon irradiation with $\mathrm{x}$-rays, the diode quantum efficiency increases with increasing polymer film thickness. The diodes produced here display characteristics similar to their thin film analogs, meaning that they may be operated in a similar way and therefore may be useful for radiation dosimetry applications. Upon irradiation, the diodes produce an x-ray photocurrent that is proportional to the dose, thus demonstrating their suitability for direct x-ray detectors. The x-ray photocurrent remains the same in a device after a cumulative exposure of $600 \mathrm{~Gy}$ and after aging for 6 months. (C) 2009 American Institute of Physics.

[doi:10.1063/1.3225909]

\section{INTRODUCTION}

Semiconducting polymers have been used over the past 20 years in emerging applications ranging from sensors to light emitting diodes (LEDs). ${ }^{1}$ Typically, these applications have involved the production of thin film diode or transistor architectures where the thickness of the active polymer film is of the order of tens to hundreds of nanometers. During the initial development of such devices, there were a number of reasons for using thin films of the semiconducting polymer, not the least of these being the need to conserve the rare and expensive polymer material. In the past 5 years, however, the polymers have become increasingly commercially available as demand increases. This availability opens the prospect of using the polymers in large scale applications, such as large area LEDs. ${ }^{2}$

Polymer availability will also open the way to the production of thick film organic diodes. Such diodes may play an important role in the production of large area radiation detectors due to their desirable characteristics ${ }^{3,4}$ (which rival those of silicon dosimeters ${ }^{5}$ ), "tissue equivalent" response to radiation, ${ }^{6}$ and large area processing possibilities. ${ }^{7}$ Emphasis has currently centered on the development of indirect radiation detectors, in which the organic photodiodes and/or transistors are coupled to phosphor screens. ${ }^{3}$ There has been little published research on the use of semiconducting polymers as

\footnotetext{
a) These authors have contributed equally to this work.

${ }^{b)}$ Authors to whom correspondence should be addressed.

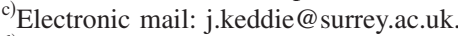

${ }^{\mathrm{d})}$ Electronic mail: p.sellin@surrey.ac.uk.
}

charge-sensitive, direct radiation detectors. ${ }^{8}$ Our group recently reported $\mathrm{x}$-ray photocurrent sensitivity measurements in semiconducting polymers, ${ }^{9}$ which suggested that useful $\mathrm{x}$-ray photocurrent sensitivity could be achieved even for relatively thin organic layers.

To be useful for charge-based radiation detection, polymeric diodes require certain attributes, including chemical and electronic stability, high carrier mobility, low leakage currents (dark current), and high current sensitivity upon exposure to radiation. The diodes would also profit by having a large thickness to increase the "capture volume" of the active material. Here, we report on the properties of a semiconducting polymer, poly(triarylamine) (PTAA) used in thick film diode structures, with the aims primarily of determining the operating conditions for the diodes and, second, of elucidating the electrical conduction mechanism occurring in the polymer.

To produce thick film diodes, the polymer must be capable of transporting charge carriers over relatively large distances to the electrodes of the device. PTAA polymers have been shown to have high charge carrier mobilities (especially when the molecular weight is low $)^{10}$ and are operationally and environmentally very stable due to a high ionization potential. ${ }^{11}$ Indeed, organic field effect transistors produced with PTAA as the semiconducting material and with a hole mobility of $10^{-3} \mathrm{~cm}^{2} \mathrm{~V}^{-1} \mathrm{~s}^{-1}$ have been used in ambient conditions. ${ }^{12}$ Other examples of triarylamine polymers have produced bulk mobilities, in time-of-flight experiments, in excess of $10^{-2} \mathrm{~cm}^{2} \mathrm{~V}^{-1} \mathrm{~s}^{-1}{ }^{13}$ The reason for this high mo- 


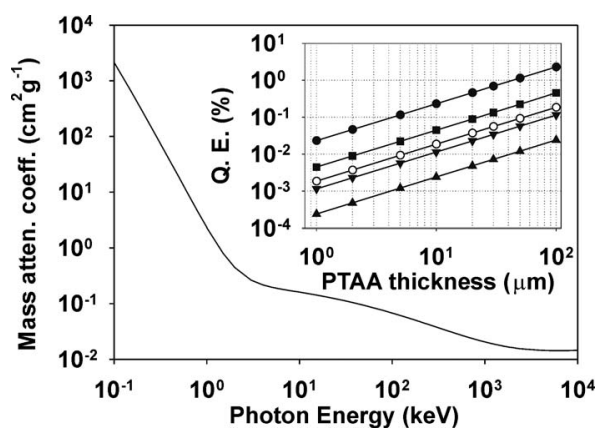

FIG. 1. Mass attenuation coefficient of the photon cross section for PTAA, calculated using XCOM. Inset: quantum efficiency (QE) for PTAA films of increasing thickness at incident $\mathrm{x}$-ray photon energies of $(\boldsymbol{O}) 10 \mathrm{keV},(\boldsymbol{\square}) 20$ $\mathrm{keV},(\bigcirc) 60 \mathrm{keV},(\boldsymbol{\nabla}) 300 \mathrm{keV}$, and (A) $6 \mathrm{MeV}$.

bility may be due to low concentrations of bulk or interface traps. Furthermore, as PTAA is a glassy polymer, there are no effects of crystal interfaces. ${ }^{14}$ PTAA polymers have previously been investigated with respect to $\mathrm{x}$-ray imaging applications by incorporation in thin film transistors. ${ }^{13}$ The devices have been shown to be stable over an equivalent device lifetime x-ray exposure dose of $500 \mathrm{~Gy}$, with no discernible degradation in device performance and no additional trap states produced. This paper investigates the suitability of PTAA for use in thick film diodes and the application of such diodes as a direct $\mathrm{x}$-ray detector.

\section{METHODS}

\section{A. Materials}

Poly(3,4-ethylenedioxythiophene)/poly(styrenesulfonate) (PEDOT:PSS) (1.3 wt \% dispersion in $\mathrm{H}_{2} \mathrm{O}$, conductive grade), and toluene $(99.99 \%)$ (both from Sigma-Aldrich Chemical Co., UK) were used as received. PTAA (inset in Fig. 2) was synthesized via a method described elsewhere ${ }^{14}$ to give a polymer with a weight-average molecular weight $\left(M_{w}\right)$ of $3.1 \times 10^{5} \mathrm{~g} \mathrm{~mol}^{-1}$ and a polydispersity index $\left(M_{w} / M_{n}\right)$ of 2.07. Indium tin oxide (ITO) coated glass, with a nominal sheet resistance of $25 \Omega$ and a deposited ITO thickness of 80-120 nm, was purchased from Delta Technology Ltd., USA (CB-60IN).

\section{B. Diode production}

For the diode fabrication, ITO glass slides were cut to size $\left(\sim 2.5 \times 2.5 \mathrm{~cm}^{2}\right)$, cleaned by rinsing in acetone, and dried under ambient conditions. PEDOT:PSS was spin-cast (PWM32, Headway Research, USA) onto the ITO giving a polymer film thickness of $\sim 150 \mathrm{~nm}$. The PEDOT:PSS was

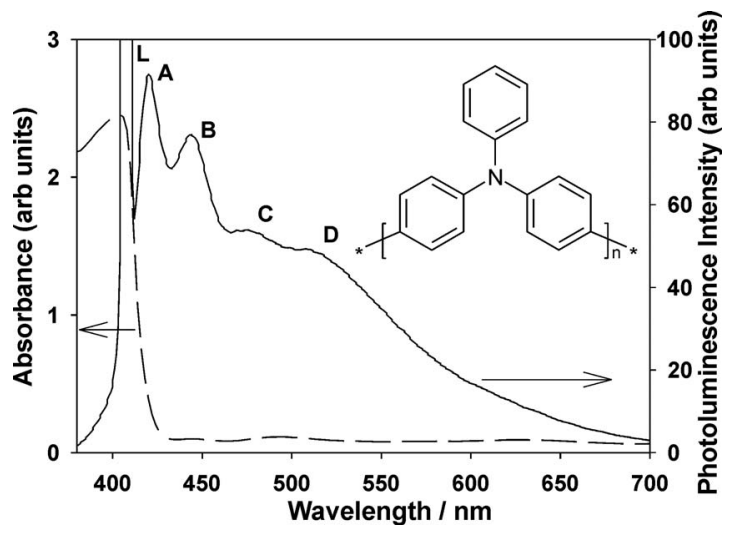

FIG. 2. Comparison of the UV-vis (dashed line) and PL (solid line) spectra of PTAA. The laser line (L) and the peaks due to structural resonance (A-D) are indicated. Also shown (inset) is the chemical structure of PTAA.

then annealed at $100{ }^{\circ} \mathrm{C}$ under vacuum for $12 \mathrm{~h}$ to eliminate any trapped solvent and was allowed to cool to room temperature under vacuum. PTAA (5 wt \% solution in toluene) was then spin-cast on top of the PEDOT:PSS layer to give various PTAA film thicknesses. The processing conditions for each of the polymer thick films are summarized in Table I. The films were allowed to dry under atmospheric conditions before annealing under vacuum at 110 or $150{ }^{\circ} \mathrm{C}$ for 12 $\mathrm{h}$. The thickness and topography of the polymer layers were subsequently measured using a surface profilometer (Dektak, Veeco Instruments).

To complete the diodes, either gold $(\mathrm{Au})$ or aluminum (Al) electrodes $\left(100 \mathrm{~nm}\right.$ thick, $\left.0.5 \times 0.5 \mathrm{~cm}^{2}\right)$ were thermally evaporated onto the PTAA through a shadow mask at a pressure of $10^{-6}$ mbar. The diodes were finally connected to the measurement electronics through filament wires attached by gold paste to the electrodes. Upon completion, the diodes were stored under nitrogen and in the dark to minimize any adverse oxidation effects.

\section{Diode characterization}

Optical absorption spectra of $1 \mu \mathrm{m}$ thick PTAA films spin-cast onto float glass were obtained using an ultravioletvisible (UV-vis) spectrometer (M350 double beam, Camspec, UK) in the wavelength range from 380 to $900 \mathrm{~nm}$. Illumination was achieved using a tungsten-halogen (370$1100 \mathrm{~nm}$ ) lamp. A complementary piece of the float glass was placed in the reference beam of the spectrometer. Room temperature photoluminescence (PL) spectra of the same samples were obtained by excitation by a blue laser $(\lambda$

TABLE I. Deposition conditions for polymer films.

\begin{tabular}{lcccccc}
\hline \hline Polymer & $\begin{array}{c}\text { Solution volume } \\
(\mathrm{mL})\end{array}$ & $\begin{array}{c}\text { Thickness } \\
(\mu \mathrm{m})\end{array}$ & $\begin{array}{c}\text { Acceleration } \\
\left(\mathrm{rpm} \mathrm{s}^{-1}\right)\end{array}$ & $\begin{array}{c}\text { Spin speed } \\
(\mathrm{rpm})\end{array}$ & $\begin{array}{c}\text { Spin time } \\
(\mathrm{s})\end{array}$ & $\begin{array}{c}\text { Deceleration } \\
\left(\mathrm{rpm} \mathrm{s}^{-1}\right)\end{array}$ \\
\hline PEDOT:PSS & 1 & 0.15 & 500 & 2000 & 30 & 500 \\
PTAA & 1 & 1 & 100 & 200 & 30 & 100 \\
PTAA & 2 & 5 & 100 & 100 & 30 & 100 \\
PTAA & 3 & 20 & 100 & 50 & 30 & 100 \\
PTAA & 5 & 30 & 100 & 50 & 30 & 100 \\
\hline \hline
\end{tabular}


$=405 \mathrm{~nm}$ ). The emission spectrum was collected in reflection geometry and was examined using a spectrometer.

The current-voltage $(I-V)$ characteristics of the ITO/ PEDOT:PSS/PTAA/metal diodes were examined using a voltage source-picoammeter (487, Keithley Instruments, UK) by applying a bias voltage from -100 to $100 \mathrm{~V}$ to the ITO electrode. Photocurrent measurements were performed using illumination from a quartz halogen lamp (100 W, LOTOriel group, UK) passed through a diffraction grating monochromator (M300, Bentham, UK). Photocurrent was recorded using the Keithley voltage source-picoammeter, interfaced to a PC controlling the monochromator, with an applied field of $10^{7} \mathrm{~V} \mathrm{~m}^{-1}$, and bias applied to the metal electrode. The photocurrent measurements were completed through diodes with semitransparent metal electrodes (thickness $=\sim 30 \mathrm{~nm}$ ). The $I-V$ and photocurrent measurements were completed in the dark, at room temperature, and under vacuum $\left(\sim 3 \times 10^{-1}\right.$ mbar $)$.

Modulated differential scanning calorimetry (MDSC) was performed on the PTAA powder using a calorimeter (Q1000, TA Instruments) in a heat-cool-heat cycle under nitrogen from 20 to $200{ }^{\circ} \mathrm{C}$ at $3{ }^{\circ} \mathrm{C} \mathrm{min}^{-1}$, modulating at $\pm 2{ }^{\circ} \mathrm{C}$ every $60 \mathrm{~s}$. Thermogravimetric analysis (TGA) was also carried out under a nitrogen atmosphere using a TGA apparatus (Q500, TA Instruments) from 20 to $300{ }^{\circ} \mathrm{C}$ with a heating rate of $5^{\circ} \mathrm{C} \mathrm{min}-1$. Dry PTAA powder and drop-cast PTAA thick films were compared.

Dosimetry experiments were conducted using $17 \mathrm{MeV}$ $K \alpha$ x-rays from a molybdenum target x-ray tube (XF50 11, Oxford Instruments, UK) operated at a $50 \mathrm{kV}$ anode voltage and a variable anode current (up to $1 \mathrm{~mA}$ ). Current-voltage measurements were recorded for the diodes under the illumination of the x-rays using the voltage source-picoammeter. Dosimetry measurements were carried out on diodes mounted in a steel box, $10 \mathrm{~cm}$ from the source, in the dark, at room temperature, and under vacuum.

\section{Quantum efficiency calculation}

The quantum efficiencies of PTAA films of various thicknesses were calculated using data obtained from a photon attenuation database. ${ }^{15}$ The mass attenuation coefficient $(\mu / \rho)$ as a function of photon energy was calculated using the equation

$$
I_{(x)}=I_{0} e^{-(\mu / \rho) \rho x},
$$

where $I_{0}$ and $I_{(x)}$ are the incident and emitted radiation intensities, respectively, and $\mu, x$, and $\rho$ are the linear attenuation coefficient, the thickness, and the density of the polymer film, respectively. For PTAA, $\rho$ was assumed to be 1 . A plot of the mass attenuation coefficient was used to find the fraction of incident photons that are absorbed for x-ray energies in the range of $10 \mathrm{keV}$ to $6 \mathrm{MeV}$. The quantum efficiencies, $\mathrm{QE}$, of the polymer films of different thicknesses, were then calculated using

$$
\mathrm{QE}=1-\frac{I_{(x)}}{I_{0}} .
$$

\section{RESULTS AND DISCUSSION}

Figure 1 shows the theoretical quantum efficiency of the PTAA films of increasing thickness. The mass attenuation coefficient of the PTAA was calculated using the molecular formula of the PTAA repeat unit $\left(\mathrm{C}_{19} \mathrm{H}_{15} \mathrm{~N}\right)$ and was obtained as the sum of the corresponding quantities for the atomic constituents. ${ }^{15}$ For $\mathrm{x}$-ray energies from 1 to $\sim 20 \mathrm{keV}$, the mass attenuation coefficient drops rapidly, with the photon interaction process dominated by photoelectric absorption. For energies between $20 \mathrm{keV}$ and $10 \mathrm{MeV}$, the mass attenuation coefficient drops less rapidly due to a combination of Compton scatter and pair production processes.

The inset figure shows the corresponding quantum efficiency for a PTAA layer, as a function of thickness, for x-ray energies of $10 \mathrm{keV}, 20 \mathrm{keV}, 60 \mathrm{keV}, 300 \mathrm{keV}$, and $6 \mathrm{MeV}$. The quantum efficiency increases linearly, over two orders of magnitude, as the PTAA thickness increases from 1 to $100 \mu \mathrm{m}$, reaching approximately $1 \%$ quantum efficiency for a $100 \mu \mathrm{m}$ thick layer at $10 \mathrm{keV}$. The ultimate objective of this work is to demonstrate the electrical and photoconductivity properties of PTAA thick film diodes under x-ray irradiation despite the relatively low quantum efficiencies provided by the pure PTAA film. Methodologies to increase the PTAA quantum efficiency by loading with high atomic number materials will be developed in future work.

Figure 2 gives an example of the UV-vis absorption and PL emission spectra for PTAA films from 380 to $700 \mathrm{~nm}$. The absorption edge (defined as the wavelength at $50 \%$ of the maximum value) is observed at $\sim 410 \mathrm{~nm}$, just above the laser line (L) at $405 \mathrm{~nm}$ in the luminescence spectrum. Also shown is the main absorption/emission wavelength for the polymer (again at $50 \%$ of the maximum value) at $\sim 410 \mathrm{~nm}$ $(\sim 3 \mathrm{eV})$, while at lower energies, a set of peaks $(\mathrm{A}-\mathrm{D})$ can be seen in the emission spectrum at $420 \mathrm{~nm}(2.95 \mathrm{eV}), 445$ $\mathrm{nm}(2.78 \mathrm{eV}), 475 \mathrm{~nm}(2.61 \mathrm{eV})$, and $510 \mathrm{~nm}(2.43 \mathrm{eV})$.

The position of the first emission peak suggests that PTAA has a band gap of $\sim 2.95 \mathrm{eV}$, which is corroborated by literature values. ${ }^{16}$ The Stokes shift $(16 \mathrm{~nm}, 0.12 \mathrm{eV})$, between the absorption edge and the PL emission spectra, is characteristic of conjugated polymers ${ }^{17}$ and is due to intrachain torsion and to intra-/interchain exciton migration occurring between absorption and emission. ${ }^{18}$ Torsional effects are expected to contribute $\sim 60-70 \mathrm{meV}$ to the Stokes shift, especially when the polymer is immobilized in the solid phase, with exciton migration accounting for the rest of the shift. ${ }^{18}$ The peaks in the PL spectrum, from 420 to $510 \mathrm{~nm}$, correspond to the vibrational progression characteristically found in conjugated polymers. ${ }^{19}$ The regular spacing of the peaks $\left(\sim 170 \mathrm{meV}, 1370 \mathrm{~cm}^{-1}\right)$ can be assigned to the $\mathrm{C}-\mathrm{C}$ stretching vibration of the phenyl groups ${ }^{20}$ or the presence of the tertiary amine in the PTAA backbone (inset in Fig. 2).

Figure 3 shows typical dc characteristics for diodes with $5 \mu \mathrm{m}$ thick PTAA layers annealed at either 110 or $150{ }^{\circ} \mathrm{C}$ and with either $\mathrm{Au}$ or $\mathrm{Al}$ top electrodes. Bias was applied to the ITO electrode in each case. Upon annealing at $110^{\circ} \mathrm{C}$, the reverse bias dark current for the ITO/PEDOT:PSS/ PTAA/Au diode is $42.4 \mathrm{nA}$ at $100 \mathrm{~V}$, with a current rectifica- 

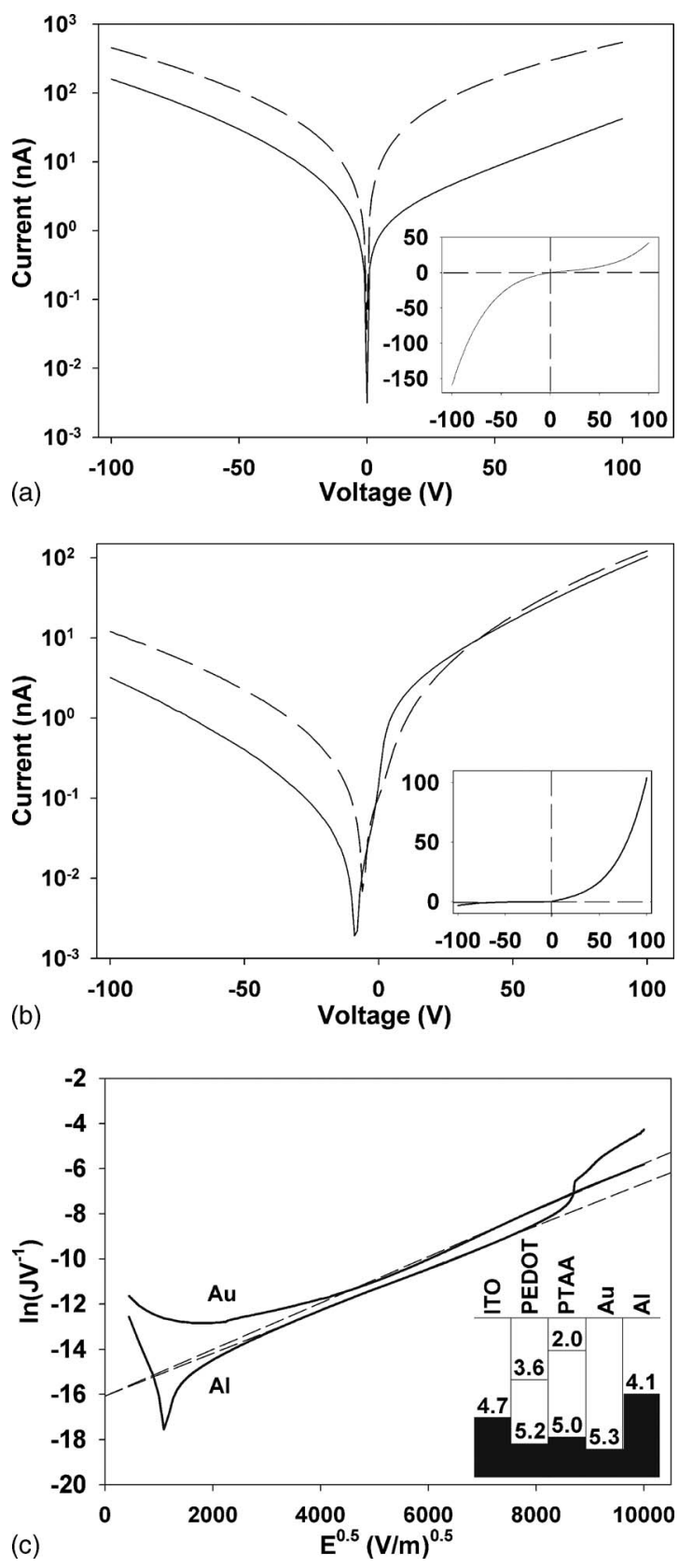

FIG. 3. Typical dc characteristics of PTAA diodes. Semilog current-voltage characteristic of (A) ITO/PEDOT:PSS/PTAA/Au and (B) ITO/ PEDOT:PSS/Al diodes with PTAA layers annealed at $110{ }^{\circ} \mathrm{C}$ (solid line) and $150{ }^{\circ} \mathrm{C}$ (dash line). Inset: Linear current-voltage plots for the $110{ }^{\circ} \mathrm{C}$ devices. (C) Fitting of the data using the Poole-Frenkel conduction model (dashed lines). Inset in (C): energy levels (in $\mathrm{eV}$ ) in the various components of the diodes.

tion factor between $\pm 100 \mathrm{~V}$ of 3.75 [Fig. 3(A)]. After annealing at the higher temperature of $150{ }^{\circ} \mathrm{C}$, the dark currents generally increase and the rectification degrades (the rectification factor at $\pm 100 \mathrm{~V}$ decreases to 0.8 ). On the other hand, the ITO/PEDOT:PSS/PTAA/Al diode [Fig. 3(B)] annealed at $110{ }^{\circ} \mathrm{C}$ displays a reverse bias dark current of $3.2 \mathrm{nA}$ at $100 \mathrm{~V}$ and a rectification factor of 32.5. Upon annealing at $150{ }^{\circ} \mathrm{C}$, the performance degrades slightly, with the reverse bias current at $100 \mathrm{~V}$ increasing to $12 \mathrm{nA}$ and the rectification factor decreasing to 10.2. Thus, the diodes with the $\mathrm{Al}$ contacts show the more desirable characteristics, with
TABLE II. Diode parameters calculated from the Poole-Frenkel plot in Fig. $3(\mathrm{C})$.

\begin{tabular}{lcc}
\hline \hline & ITO/PEDOT:PSS/PTTA/Au & ITO/PEDOT:PSS/PTAA/Al \\
\hline$E\left(\mathrm{~V} \mathrm{~m}^{-1}\right)$ & $1.6 \times 10^{7}<E<1 \times 10^{8}$ & $9 \times 10^{6}<E<6.4 \times 10^{7}$ \\
$r^{2}$ & 0.9978 & 0.9994 \\
$\varepsilon_{r}$ & 8.26 & 9.85 \\
$\phi_{\mathrm{PF}}(\mathrm{eV})$ & 0.422 & 0.406 \\
\hline \hline
\end{tabular}

a lower dark current and a higher rectification and with the lower temperature anneal as the optimum process. Diodes with thicker PTAA layers $(20$ and $30 \mu \mathrm{m})$ show similar $I-V$ characteristics although with smaller current values.

The dc electrical characteristics of the $5 \mu \mathrm{m}$ thick PTAA diodes have been examined with respect to a number of conduction models, including Schottky, ${ }^{21}$ Poole-Frenkel, ${ }^{22}$ Fowler-Nordheim, ${ }^{23}$ and space charge limited current ${ }^{24}$ models, with the aim of elucidating the charge carrier conduction mechanism. Within the typical voltage range of these devices, the mean field strength, $E$, at $100 \mathrm{~V}$ corresponds to $2 \times 10^{7} \mathrm{~V} \mathrm{~m}^{-1}$. In this field regime, the the Poole-Frenkel mechanism satisfactorily describes the observed conduction, as shown in Fig. 3(C). The Poole-Frenkel mechanism is a bulk dielectric effect that describes the lowering of potential energy wells in the semiconductor when $E>10^{7} \mathrm{~V} \mathrm{~m}^{-1}{ }^{22}$

By the application of the Poole-Frenkel theory to the data in Fig. 3, the relative permittivity, $\varepsilon_{r}$, and the respective potential barrier heights, $\phi$, can be calculated (Table II). The calculated permittivity value of $\varepsilon_{r} \approx 9$ is higher than other $p$-type semiconducting polymers used in diode applications, e.g., PCDM (Ref. 25) $\varepsilon_{r} \approx 7$ and P3MeT (Ref. 26) $\varepsilon_{r} \approx 5$, but within the range expected for common electroactive polymers $\left(2>\varepsilon_{r}>12\right) .{ }^{27}$ The height of the potential wells in the bulk of the PTAA $\left(\phi_{\mathrm{PF}}\right)$ is found to be $\sim 0.41 \mathrm{eV}$, irrespective of the electrode metal used. For comparison, this mechanism has previously been used to explain conduction in an $\mathrm{Al} /$ acid-doped poly(aniline)/Al device at high fields. ${ }^{28}$

Using literature values for PEDOT:PSS, ${ }^{29}$ ITO, and the metals, $\mathrm{Al}$ and $\mathrm{Au},{ }^{30}$ the relative positions of the energy levels in the PTAA diodes can be plotted [inset in Fig. 3(C)]. The band gap of the PTAA, $E_{g}(\approx 3 \mathrm{eV})$, is given by the optical spectroscopy (Fig. 2) and is in line with literature values for similar polymers. ${ }^{31}$ The position of the PTAA highest occupied molecular orbital (HOMO), with respect to the metal contacts, can be taken from the literature. ${ }^{16}$ The $I-V$ curves for the ITO/PEDOT:PSS/PTAA/Au device suggest that the Au contact does not make a good rectifying contact to the PTAA. Rather, the polymer typically forms Ohmic contacts with both electrodes probably due to the similarity between the energies of the Au work function and the PEDOT:PSS HOMO. This explains why the diode displays very low rectification values and a switch in the position of the low current regime (dark current) upon annealing [Fig. $3(\mathrm{~A})$. $\mathrm{Al}$, because of the greater difference between the energies of its work function and the HOMO of the PTAA, produces a good rectifying contact with the polymer, as evidenced in Fig. 3(B). The current-voltage characteristics sug- 

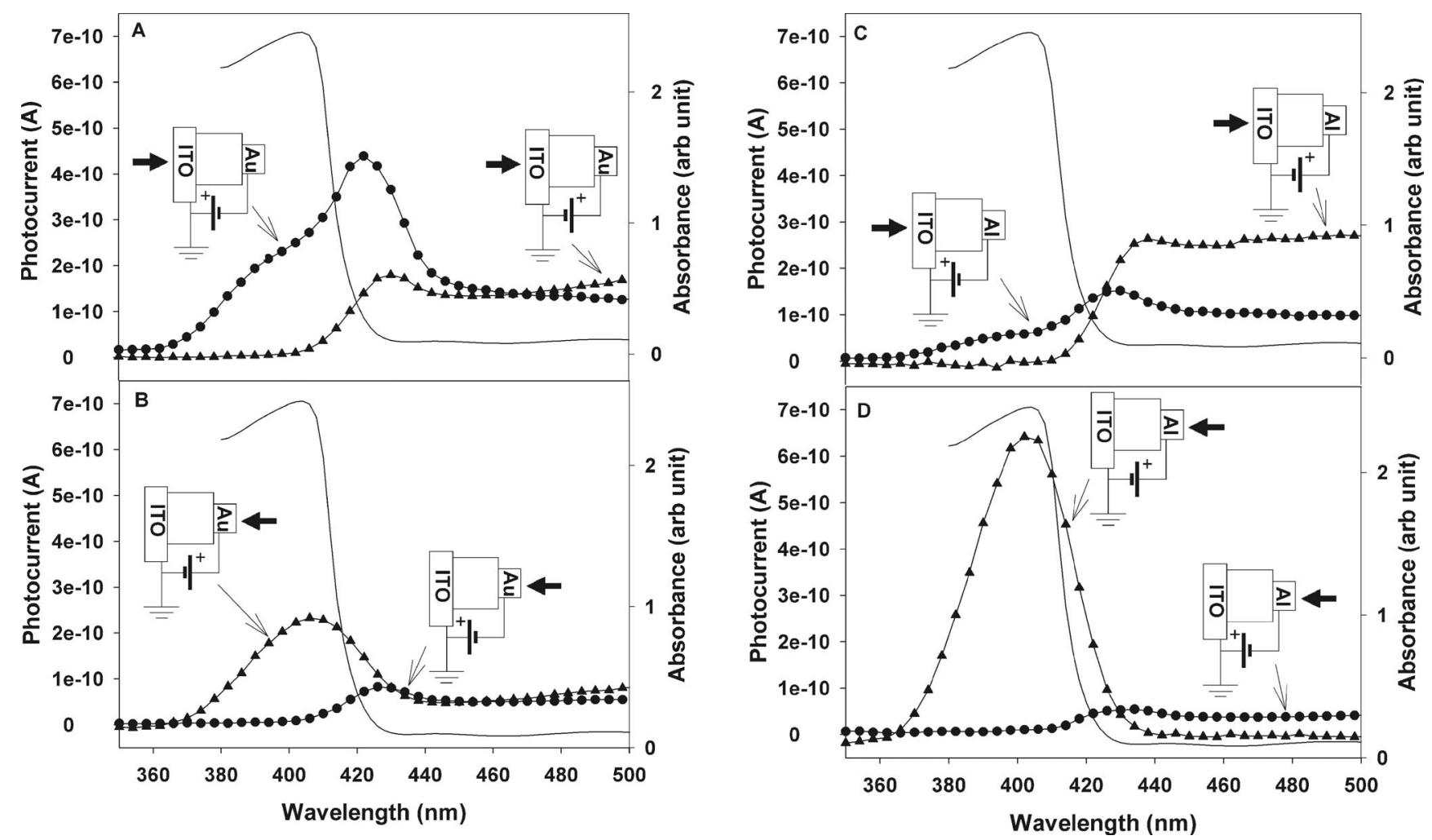

FIG. 4. Comparison of the UV-vis (solid line) and optical photocurrent spectra for ITO/PTAA/metal diodes: with gold electrodes, with illumination through (A) the ITO and (B) the Au, and with aluminum electrodes, with illumination through the ITO (C) and the Al (D). In each case, the ITO electrode is biased positively $(\bullet)$ and negatively $(\mathbf{\Lambda})$.

gest that a depletion layer is formed at the $\mathrm{Al}$ electrode in the ITO/PEDOT:PSS/PTAA/Al diode, which leads to the low current regime in reverse bias, and this, allied with the photocurrent results, suggests that PTAA is a $p$-type polymer.

The optically excited photocurrent and UV-vis absorption spectra of ITO/PTAA/metal diodes are plotted in Fig. 4 for various bias polarity and illumination combinations. A comparison of the spectra shows that at wavelengths near the band edge, 380-420 nm, the plots display a symbatic/ antibatic relationship: That is, generally, when the photocurrent is at a maximum, the absorption is at a minimum, and vice versa. ${ }^{32}$ This effect has been seen previously in semiconducting polymer diodes ${ }^{33}$ and can be used to confirm the semiconducting polymer type ${ }^{34}$ depending on the bias polarity and the electrode face exposed to the exciting light. In general, the photocurrent amplitude is maximized when photons of energy close to the band edge are incident on a junction that collects the less mobile "minority" charge carrier and at which the local field strength is greatest. With reference to Fig. 3(B), where the bias is applied to the ITO electrode, the maximized local field strength occurs in our devices when the Al/PTAA junction is in reverse bias, which corresponds to the Al/PTAA junction biased as the anode. From the data in Fig. 3(A), the position of the maximized local field is less clear due to the uncertainty as to the position of the depletion layer in the diode (as evidenced by the switch in the polarity of the dark current regime upon heating at 110 and $150{ }^{\circ} \mathrm{C}$ ).

In contrast, the photocurrent spectra in Fig. 4 show the diodes with the bias applied to the metal electrode. Figures
4(A) and 4(B) show the data for the Au/PTAA device, where the larger photocurrent peak $(\sim 405 \mathrm{~nm})$ is seen when the anode is illuminated in each case. For the Al/PTAA device, the photocurrent is particularly strong when the reversebiased Al/PTAA junction is illuminated, as shown in Fig. 4(D). Again, in this case, the Al/PTAA junction acts as the anode, and the local field is maximized at this electrode. The photocurrent reaches a maximum value at a wavelength of $\sim 405 \mathrm{~nm}$, corresponding to the absorption of the optical photon in the near-surface region of the junction where the field strength is maximum and is consistent with a good hole transport through the bulk of the layer. Forward biasing the diode minimizes the field at the $\mathrm{Al}$ electrode, resulting in low photocurrents. These data confirm the superiority of $\mathrm{Al}$ as the metallic contact for the production of photocurrent from these PTAA diode structures, compared to Au.

The increase in current produced upon annealing the PTAA (Fig. 3) may be explained by examining the thermal characteristics of the polymer (Fig. 5). MDSC shows a change in heat capacity at around $102{ }^{\circ} \mathrm{C}$, which is assigned to the glass transition temperature $\left(T_{g}\right)$ of the PTAA [Fig. 5(A)]. This $T_{g}$ value is close to the value of $98{ }^{\circ} \mathrm{C}$ obtained elsewhere ${ }^{10}$ through a dynamic mechanical analysis of PTAA with a $M_{w} \approx 10^{5} \mathrm{~g} \mathrm{~mol}^{-1}$ (which is similar to the $M_{w}$ used here). No melting or other first-order phase transitions can be seen in the differential scanning calorimetry thermogram [inset in Fig. 5(A)], revealing that PTAA is an amorphous polymer. 

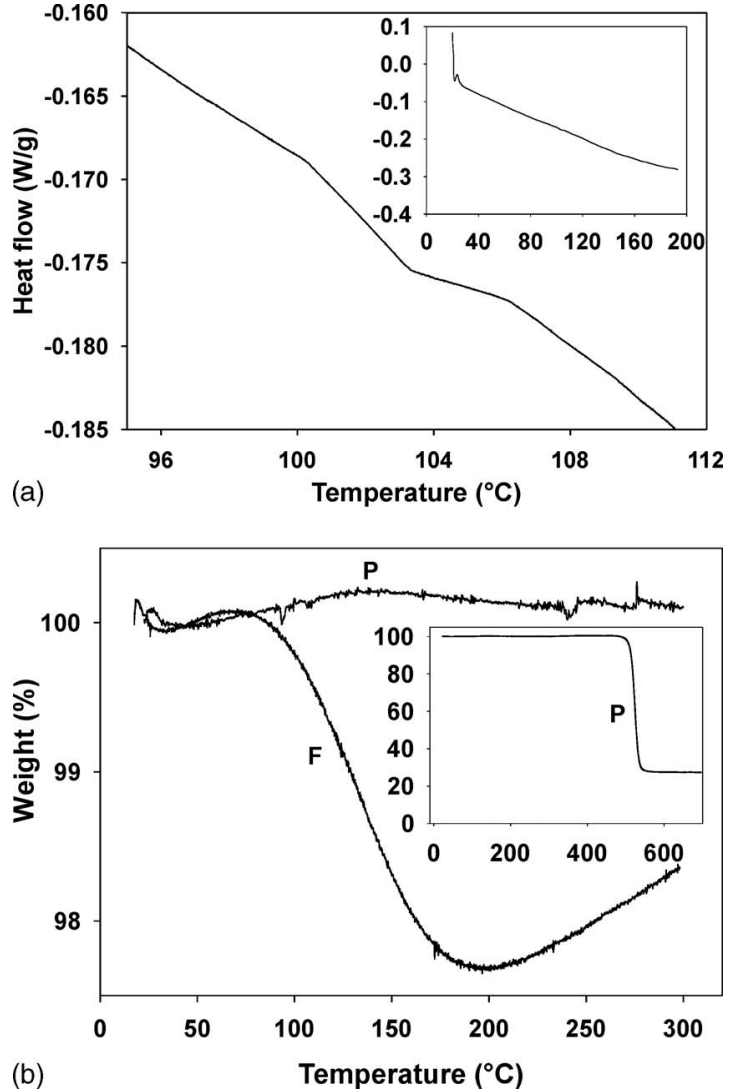

FIG. 5. Thermal analysis of PTAA. (A) MDSC thermogram (inset: full range) and (B) thermogravimetric analysis of (P) dry PTAA powder and (F) freshly cast PTAA thick film (inset: full range for P).

In previous experimental ${ }^{35}$ and theoretical ${ }^{36}$ studies of solvent-cast glassy polymer films, it has been found that the rigid glass structure inhibits solvent loss. Solvent loss and the accompanying structural relaxation are exceedingly slow in glassy films at sub- $T_{g}$ temperatures. ${ }^{37}$ In the preparation of the diodes used here, the PTAA was annealed above its $T_{g}$ to allow any solvent trapped inside the film to escape. To examine the solvent loss from the PTAA films, TGA was employed [Fig. 5(B)]. The thermogram for dry PTAA powder shows that no significant weight loss occurs up to $500{ }^{\circ} \mathrm{C}$ (see the inset), at which point a $70 \%$ weight loss is observed which may be ascribed to the thermal decomposition of the PTAA. For a thick solvent-cast PTAA film, by comparison, a reduction in weight of $\sim 2.1 \%$ is seen between 100 and $200{ }^{\circ} \mathrm{C}$, which is attributed to the liberation of the toluene solvent used in the film deposition. When the heating temperature exceeds the $T_{g}$ of the PTAA, the polymer is able to relax and to enable the liberation of the toluene. ${ }^{36,37}$ At $\sim 200{ }^{\circ} \mathrm{C}$, the weight is stable, which indicates that toluene is completely removed; however, the data indicate that the rate of solvent loss is significantly slower near $110^{\circ} \mathrm{C}$.

These TGA results explain the change in the electrical properties upon annealing. Solvent residues present in the film, which are only partially removed at $110^{\circ} \mathrm{C}$, act as trapping sites for charge carriers. After annealing at $150{ }^{\circ} \mathrm{C}$, however, the solvent in the diode films is eliminated. Small traces of contaminants, such as solvent molecules, can affect the properties of semiconducting diodes. ${ }^{38}$ Toluene in this

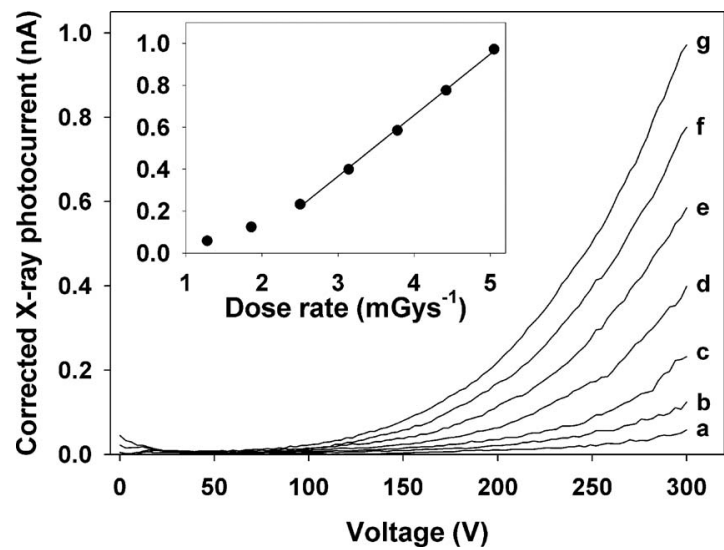

FIG. 6. Corrected x-ray photocurrent vs applied voltage at increasing dose rates for a reverse-biased ITO/PEDOT:PSS/PTAA/Al diode with a $30 \mu \mathrm{m}$ thick PTAA layer annealed at $150{ }^{\circ} \mathrm{C}$ and irradiated through the ITO electrode. Dose rates: (a) $1.28 \mathrm{mGy} \mathrm{s}^{-1}$, (b) $1.86 \mathrm{mGy} \mathrm{s}^{-1}$, (c) $2.50 \mathrm{mGy} \mathrm{s}^{-1}$, (d) $3.14 \mathrm{mGy} \mathrm{s}^{-1}$, (e) $3.78 \mathrm{mGy} \mathrm{s}^{-1}$, (f) $4.42 \mathrm{mGy} \mathrm{s}^{-1}$, and (g) $5.05 \mathrm{mGy} \mathrm{s}^{-1}$. Inset: corrected $\mathrm{x}$-ray photocurrent vs $\mathrm{x}$-ray dose rate at $-300 \mathrm{~V}$ applied operating voltage. Data in both cases are inverted for clarity.

case may act to trap charge carriers produced on the semiconducting PTAA. Removing solvent from the film enables charge carriers to move across the polymer more efficiently and hence to increase the current produced by the diode. When considering the requirements for the dosimeters, however, this increase in conduction must be offset by the requirement for a low dark current.

The $\mathrm{x}$-ray sensitivity of the PTAA diode was measured in terms of x-ray photocurrent using a $17 \mathrm{kV}$ (peak) $\mathrm{x}$-ray generator. The ITO contact of a reverse-biased ITO/ PEDOT:PSS/PTAA/Al diode, with bias applied to the ITO electrode, was irradiated with $\mathrm{x}$-rays as a function of increasing applied electric field, at seven different dose rates in the range from 1.28 to $5.05 \mathrm{mGy} \mathrm{s}^{-1}$. Figure 6 shows the recorded $\mathrm{x}$-ray induced photocurrents after the subtraction of the device dark current as the voltage is increased from 0 to $300 \mathrm{~V}$ for each $\mathrm{x}$-ray dose. The inset figure shows the resulting $\mathrm{x}$-ray photocurrent as a function of the dose rate, recorded at a $300 \mathrm{~V}$ reverse bias on the device. The data show an initial growth in the photocurrent at low dose rates, followed by a region of linear response at dose rates above $\sim 2.5 \mathrm{mGy} \mathrm{s}^{-1}$, with a calculated detection sensitivity of $\sim 0.3 \mathrm{nC} / \mathrm{mGy}$.

Finally, the stability of the response of an ITO/ PEDOT:PSS/PTAA/Au diode annealed at $150{ }^{\circ} \mathrm{C}$ and operated at an electric field of $5 \times 10^{4} \mathrm{~V} / \mathrm{cm}(150 \mathrm{~V})$ is shown in Fig. 7. The graph shows two photocurrent measurements at increasing dose rates, taken 6 months apart, on a diode that was used repeatedly during that time and exposed to an estimated accumulated dose of $\sim 600 \mathrm{~Gy}$. The standard deviation of the data points at each dose rate is less than $0.1 \mathrm{nA}$, showing that the diodes have good long-term storage stability. Furthermore, there is no evidence that repeated radiation doses degrade the device's performance.

\section{CONCLUSIONS}

PTAA thick film polymer diodes are shown to operate in a similar way to analogous thin film semiconductor devices 


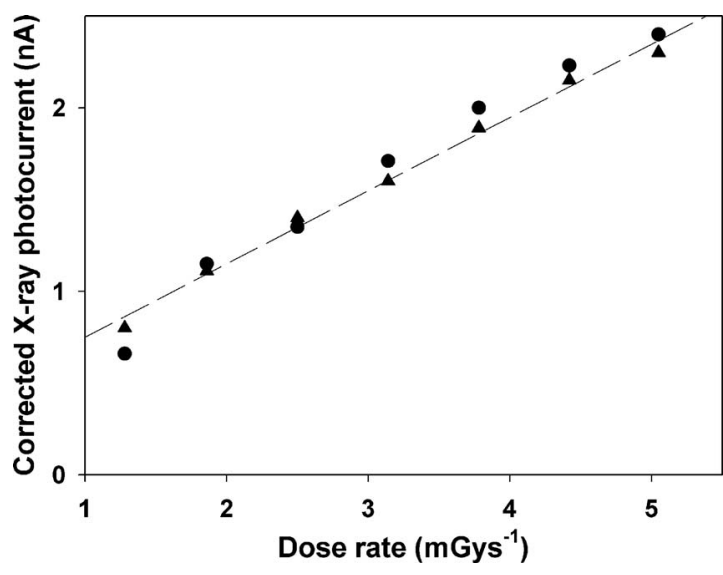

FIG. 7. X-ray photocurrent vs applied dose rate measured (๑) after completion of the diode and ( $\mathbf{\Delta}) 6$ months later for a reverse-biased ITO/ PEDOT:PSS/PTAA/Au diode with a $30 \mu \mathrm{m}$ thick PTAA layer. The dashed line is the regression line for all the data in the plot $\left(r^{2}=0.9926\right)$.

and display a bulk dielectric conduction mechanism. PTAA diodes with $\mathrm{Al}$ contacts have stable low leakage currents, high field strength, and good rectification performance. Such diodes were shown to detect incident $\mathrm{x}$-ray irradiation, exhibiting a linear relationship between the dose and the x-ray photocurrent and exhibiting stability after a radiation dose of 600 Gy and 6 months of aging. Thick PTAA films absorb a sufficient amount of radiation within the active area of the detector, which in turn produces sufficient charge carriers for detection. Hence, the devices are envisioned to have a number of applications in charge-based radiation detection. An understanding of the charge-based detection mechanism opens the possibility of real-time radiation dosimetry, similar to that found in analogous silicon dosimeters. ${ }^{39}$ PTAA x-ray detectors are expected to retain the mechanical flexibility and ease of processing that is typical of polymer devices and thus offer an attractive alternative to conventional semiconductor detectors.

\section{ACKNOWLEDGMENTS}

A.I. acknowledges a scholarship from the Royal Thai government. The authors acknowledge financial support from the Science and Technology Facilities Council (STFC) (Grant No. ST/F006667/1). The authors thank Dr. Veeramani Perumal, Gary Strudwick, and Violeta Dukova for help with diode preparation and characterization (all from the Department of Physics, University of Surrey) and Professor Andrew Nisbet (Department of Medical Physics, The Royal Surrey County Hospital) and Professor D. Martin Taylor (Bangor University, Wales) for helpful discussions.

${ }^{1}$ Y.-L. Loo and I. McCulloch, MRS Bull. 33, 653 (2008).

${ }^{2}$ R. E. Gill, P. van de Weijer, C. T. H. Liedenbaum, H. F. M. Schoo, A. Berntsen, J. J. M. Vleggaar, and R. J. Visser, Opt. Mater. (Amsterdam, Neth.) 12, 183 (1999).

${ }^{3}$ J. C. Blakesley, P. E. Keivanidis, M. Campoy-Quiles, C. R. Newman, Y. Jin, R. Speller, H. Sirringhaus, N. C. Greenham, J. Nelson, and P. Stavrinou, Nucl. Instrum. Methods Phys. Res. A 580, 774 (2007).
${ }^{4}$ C. J. Wong, T. Ackerly, C. He, W. Patterson, C. E. Powell, A. Ho, G. Qiao, D. H. Solomon, R. Meder, and M. Geso, Appl. Radiat. Isot. 65, 1160 (2007); S. J. Doran, ibid. 67, 393 (2009).

${ }^{5}$ K. Jordan, J. Phys.: Conf. Ser. 56, 132 (2006); Q. Ma, S. Tang, J. Zou, and D. Xu, Nucl. Sci. Techniques 19, 50 (2008).

${ }^{6}$ B. D. Milbrath, A. J. Peurrung, M. Bliss, and W. J. Weber, J. Mater. Res. 23, 2561 (2008)

${ }^{7}$ P. Sellakumar, E. J. J. Samuel, and S. S. Supe, Radiat. Phys. Chem. 76, 1108 (2007)

${ }^{8}$ P. Beckerle and H. Strobele, Nucl. Instrum. Methods Phys. Res. A 449, 302 (2000); Y. Wang and N. Herron, Science 273, 632 (1996); D. Natali and M. Sampietro, Nucl. Instrum. Methods Phys. Res. A 512, 419 (2003).

${ }^{9}$ F. D. Boroumand, M. Zhu, A. B. Dalton, J. L. Keddie, P. J. Sellin, and J. J. Gutierrez, Appl. Phys. Lett. 91, 033509 (2007).

${ }^{10}$ S. Barard, M. Heeney, L. Chen, M. Cölle, M. Shkunov, I. McCulloch, N. Stingelin, M. Philips, and T. Kreouzis, J. Appl. Phys. 105, 013701 (2009).

${ }^{11}$ H. Sirringhaus, Adv. Mater. 17, 2411 (2005).

${ }^{12}$ J. Veres, S. Ogier, and G. Lloyd, Chem. Mater. 16, 4543 (2004).

${ }^{13}$ C. R. Newman, H. Sirringhaus, J. C. Blakesley, and R. Speller, Appl. Phys. Lett. 91, 142105 (2007)

${ }^{14}$ T. Yamamoto, T. Ito, and K. Kubota, Chem. Lett. 17, 153 (1988).

${ }^{15}$ M. J. Berger, J. H. Hubbell, S. M. Seltzer, J. Chang, J. S. Coursey, R. Sukumar, and D. S. Zucker, XCOM, Photon Cross Sections Database, National Institute of Standards and Technology, USA, Standard Reference Database 8 (XGAM) (http://physics.nist.gov/PhysRefData/Xcom/Text/ XCOM.html)

${ }^{16}$ B. R. Saunders and M. L. Turner, Adv. Colloid Interface Sci. 138, 1 (2008).

${ }^{17}$ K. Kaeriyama, Y. Tsukahara, S. Negoro, N. Tanigaki, and H. Masuda, Synth. Met. 84, 263 (1997)

${ }^{18}$ K. Kanemoto, T. Sudo, I. Akai, H. Hashimoto, T. Karasawa, Y. Aso, and T. Otsubo, Phys. Rev. B 73, 235203 (2006).

${ }^{19}$ L. O. Peres, S. H. Wang, J. Wery, G. Froyer, and E. Faulques, Mater. Sci. Eng., C 29, 372 (2009).

${ }^{20}$ A. P. Monkman, M. Halim, I. D. W. Samuel, and L. E. Horsburgh, J. Chem. Phys. 109, 10372 (1998).

${ }^{21}$ D. M. Taylor, IEEE Trans. Dielectr. Electr. Insul. 13, 1063 (2006).

${ }^{22}$ J. Frenkel, Phys. Rev. 54, 647 (1938).

${ }^{23}$ A. J. Heeger, I. D. Parker, and Y. Yang, Synth. Met. 67, 23 (1994).

${ }^{24}$ M. A. Lampert, Phys. Rev. 103, 1648 (1956); P. Mark and W. Helfrich, J. Appl. Phys. 33, 205 (1962).

${ }^{25}$ C. A. Mills, D. M. Taylor, A. Riul, and A. P. Lee, J. Appl. Phys. 91, 5182 (2002).

${ }^{26}$ D. M. Taylor and H. L. Gomes, J. Phys. D 28, 2554 (1995).

${ }^{27}$ Q.-D. Ling, D.-J. Liaw, C. Zhu, D. S.-H. Chan, E.-T. Kang, and K.-G. Neoh, Prog. Polym. Sci. 33, 917 (2008).

${ }^{28}$ S. Sakthivel, B. Chandar Shekar, D. Mangalaraj, S. K. Narayandass, S. Venkatachalam, and P. V. Prabhakaran, Eur. Polym. J. 33, 1747 (1997).

${ }^{29}$ Manufacturers (Sigma-Aldrich) band-gap estimate: $1.6 \mathrm{eV}$ (www.sigmaaldrich.co.uk).

${ }^{30}$ G. Bernardo, A. Charas, L. Alcácer, and J. Morgado, J. Appl. Phys. 103, 084510 (2008).

${ }^{31}$ J. H. Sim, K. Yamada, S. H. Lee, S. Yokokura, and H. Sato, Synth. Met. 157, 940 (2007).

${ }^{32}$ M. G. Harrison and J. Grüner, Synth. Met. 84, 653 (1997).

${ }^{33}$ M. G. Harrison, J. Grüner, and G. C. W. Spencer, Phys. Rev. B 55, 7831 (1997); K. Pichler, D. A. Halliday, D. D. C. Bradley, P. L. Bum, R. H. Friend, and A. B. Holmes, J. Phys.: Condens. Matter 5, 7155 (1993).

${ }^{34}$ F. Feller and A. P. Monkman, Appl. Phys. Lett. 76, 664 (2000).

${ }^{35}$ H. Richardson, M. Sferrazza, and J. L. Keddie, Eur. Phys. J. E 12, 87 (2003).

${ }^{36}$ S. G. Croll, J. Appl. Polym. Sci. 23, 847 (1979); G. Reiter and P.-G. de Gennes, Eur. Phys. J. E 6, 25 (2001).

${ }^{37}$ H. Richardson, Í. López-García, M. Sferrazza, and J. L. Keddie, Phys. Rev. E 70, 051805 (2004)

${ }^{38}$ D. R. Baigent, F. Cacialli, N. C. Greenham, J. Gruner, H. F. Wittmann, R. H. Friend, S. C. Moratti, and A. B. Holmes, Solid-State Electron. 40, 477 (1996).

${ }^{39}$ B. G. Lowe and R. A. Sareen, Nucl. Instrum. Methods Phys. Res. A 576, 367 (2007). 